\title{
Zur sozialen Solidarität in der Gesundheitsversicherung ${ }^{1}$
}

\author{
Daniel Eryk Lach
}

\section{Einführung - Bedeutung des Grundsatzes der sozialen Solidarität für den Aufbau des Systems der Gesundheitsfürsorge}

Geradezu offensichtlich erscheint die These, dass der Grundsatz der sozialen Solidarität ein Fundament (einen Leitgedanken) für den versicherungsbasierten Ansatz zur Lösung der sozialen Absicherung darstellt. ${ }^{2}$ Aussagen dieser Art lassen sich sowohl der Literatur ${ }^{3}$ als auch der Rechtsprechung des Verfassungsgerichtshofs ${ }^{4}$ entnehmen. In Bezug auf die Gesundheitsfürsorge sieht Art. 65 des Gesetzes vom 27. August 2004 über Leistungen der aus öffentlichen Mitteln finanzierter Gesundheitsfürsorge ${ }^{5}$ (im Folgenden: LÖffGG) die Solidarität explizit als einen Leitgrundsatz für

1 Es handelt sich hierbei um eine abgeänderte und ergänzte Fassung des Vortrags von der XIX. Tagung von Lehrstühlen für Arbeits- und Sozialversicherungsrecht unter dem Titel „Axiologische Grundlagen des Arbeits- und Sozialversicherungsrechts“, Posen, 15.-17. Mai 2013, veröffentlicht in: Skapski/Ślebzak (Hrsg.), Aksjologiczne podstawy prawa pracy i ubezpieczeń społecznych, Posen 2014, S. 271-285.

2 Es ist auch die Bedeutung der Solidarität als ein allgemeines Prinzip der Unionsrechtsordnung zu bemerken. Becker betonte dazu, dass die Solidarität nicht unmittelbar zur Herleitung ungeschriebener Rechte und Pflichten dient, denn sie besitzt auch als Prinzip vorrechtlichen Charakter und bedarf immer der Konkretisierung durch gesetzliche Akte, wenn aus ihr verbindliche Folgen abgeleitet werden sollen. Das gilt gerade auch für die mit sozialen Interventionen verbundene Umverteilung, für die Solidarität ein Leitbegriff ist. Nichtsdestotrotz hängt die Legitimität der Rechtsordnung auch davon ab, dass sie unter den Bürgern Solidarität im Sinne der Sicherung der eigenen Grundlagen - auf nationaler wie auf europäischer Ebene herstellt und bewahrt. Vgl. Becker, Sozialstaatlichkeit in der Europäischen Union, in: Hatje (Hrsg.), Verfassungszustand und Verfassungsentwicklung in der Europäischen Union, EuR Beiheft. 2/2015, S. 36.

3 So Jończyk, Prawo zabezpieczenia społecznego, Krakau 2001, S. 38.

4 Vgl. VerfGH v. 11.02.1992, K 14/91, OTK 1992, H. 1, Pos. 7.

5 Konsolidierte Fassung: Poln. GB1. 2008, Nr. 164, Pos. 1027 mit späteren Änderungen. 
die Organisation der „Gesundheitsversicherung“6 vor. Dabei ist die Annahme zulässig, es handele sich nicht lediglich um einen starren, beschreibenden Grundsatz (die Bestimmung, wie gewisse Rechtsinstitute der „Gesundheitsversicherung" beschaffen sein sollen), sondern ebenfalls um einen leitenden Grundsatz i.S. einer bestimmten Verhaltensvorgabe. Der vom Gesetzgeber für Art. 65 LÖffGG gewählte Wortlaut, die Gesundheitsversicherung, ,werde nach dem Grundsatz der sozialen Solidarität verwirklicht", lässt die Annahme zu, dass diesem eine besondere Rolle im Aufbau der öffentlich finanzierten Gesundheitsfürsorge zugeschrieben wurde. Somit bildet er nicht nur einen Ansatzpunkt für sozialpolitische Wertungen, sondern stellt vor allem eine materielle Begründung für eine Reihe von Normen, insbesondere zur Finanzierung des Systems, dar. ${ }^{7}$

Der Grundsatz der sozialen Solidarität bildete auch die Grundlage für Lösungen wie der beitragsfreien Mitversicherung von Familienmitgliedern, dem Absehen von einer Bindung der garantierten Leistungen an die Beitragshöhe sowie der uneingeschränkten Höhe der Beitragsbemessungs-

6 Der Begriff „Gesundheitsversicherung“ wird nachfolgend in Anführungszeichen angegeben, da das System der Gesundheitsfürsorge, entgegen seiner Bezeichnung als Versicherung, nicht nach versicherungstechnischen Grundsätzen aufgebaut ist. Der Nationale Gesundheitsfonds - weder ein Versicherer im eigentlichen Sinne des Wortes noch ein staatliches Organ - fungiert als Verwalter für öffentliche, größtenteils beitragsfinanzierte Mittel und nimmt im Wesentlichen die Aufgaben eines Treuhänders der Leistungsempfänger wahr. Er handelt nämlich im eigenen Namen (z.B. bei Vertragsschlüssen mit Leistungserbringern), gleichwohl aber auch im Interesse der Leistungsempfänger (weil sie letzten Endes die Anspruchsberechtigten aus den vertraglich gesicherten Leistungen sind). Trotz der Versicherungsstaffage und der Zahlung von „Beiträgen“ genießen die „Versicherten“ keine körperschaftlichen Rechte gegenüber dem Nationalen Gesundheitsfonds. Dieser ist zwar eine speziell für die Verwaltung und den Betrieb vom Gesundheitswesen ins Leben gerufener professioneller Treuhänder, jedoch gerade keine selbstverwaltende, zur Vertretung der Versichertengemeinschaft berufene Versicherungsinstitution. Wenngleich er im Interesse der Leistungsempfänger handelt, so tut er dies dennoch im eigenen Namen als eigenständiger Rechtsträger mit einem gesetzlich genau bestimmten Kompetenzbereich. Vertiefend: Lach, Powiernicza technika realizacji zabezpieczenia społecznego (na przykładzie ubezpieczenia zdrowotnego), Państwo i Prawo 2009, Heft 3, S. 34-47.

7 Zum begrifflichen Verständnis von Rechtsgrundsätzen vgl. Wronkowska/Zieliński/ Ziembiński, Zasady prawa. Zagadnienia podstawowe, Warschau 1974, S. 24 ff.; Wróblewski, Prawo obowiązujące a „ogólne zasady prawa“, Zeszyty Naukowe Uniwersytetu Łódzkiego 1965, Heft 42, S. 18 ff., aber auch: Opałek/Wróblewski, Zagadnienia teorii prawa, Warschau 1969, S. 92 ff. 
grundlage und dem Heranziehen aller Einkommensquellen bei ihrer Festsetzung (Grundsatz des universalen Beitrags). Gleichzeitig liefert er auch eine Begründung für die aus diesem Grundsatz resultierende Ungleichbehandlung verschiedener Gruppen von Leistungsempfängern bei der Regelung ihrer Versicherungsverhältnisse. Dies ist insbesondere für das in Art. 68 Abs. 2 der polnischen Verfassung aufgestellte Gebot der Schaffung eines Systems der Gesundheitsfürsorge von Bedeutung, das nicht nur der praktischen Umsetzung des Rechts auf Leben und dem damit verbundenen Recht auf den Schutz der eigenen Gesundheit dienen soll, sondern überdies den Bürgern unabhängig von ihrer Einkommenslage einen gleichen Zugang zu Leistungen der öffentlich finanzierten Gesundheitsfürsorge im Krankheitsfall zu gewährleisten hat.

Bevor zu weiteren Überlegungen übergegangen werden kann, gilt es zu betonen, dass der Grundsatz der sozialen Solidarität hauptsächlich die Pflichten der Leistungsempfänger, nicht hingegen ihre Rechte betrifft. Anders ausgedrückt: Durch den Grundsatz der sozialen Solidarität sind die Teilhabe an der Risikogemeinschaft und der Umfang der Verpflichtung zur Lastentragung zu betrachten. Während die Leistungsberechtigung bereits auf die bloße Teilhabe zurückzuführen ist, bleibt der Umfang der Berechtigung von der individuellen Bedürftigkeit abhängig - der besagte Grundsatz findet demnach an dieser Stelle keine Anwendung.

Ein kurzer Blick auf diese Problematik zeigt, dass die für das Privatrecht bezeichnende Gegenseitigkeit von Beitrag und Anspruch auf Leistungen gerade keine Geltung für das aktuelle Gewährleistungssystem der Gesundheitsfürsorge beansprucht. Entscheidend hierfür ist, dass die Leistungsberechtigung (die Teilhabe an der gesetzlichen Leistungsgarantie) und die Pflicht zur Zahlung von Beiträgen in keinem zwingenden Verhältnis zueinander stehen. Einen Hinweis dafür bietet Art. 67 LÖffGG. Der Gesetzgeber unterscheidet zwischen der „Erfüllung der Versicherungspflicht" (Abs. 1) durch Anmeldung und die Entrichtung der eigenen Beiträge einerseits und dem „Erwerb von Leistungsrechten im System der Gesundheitsfürsorge" andererseits, für den bereits die Erfüllung der entsprechenden Anmeldepflichten ausreichend ist (Abs. 2). Daraus lässt sich ableiten, dass auch wenn die Zahlung von Versicherungsbeiträgen im System der Gesundheitsfürsorge zu den grundlegenden Pflichten der Leistungsempfänger gehört, der Erwerb von Leistungsansprüchen dennoch ausschließlich von der Anmeldung im dafür vorgesehenen Verfahren ab- 
hängig ist. ${ }^{8}$ Offensichtlich ausgeschlossen ist aus diesem Grund die Gegenseitigkeit der besagten Leistungen - des Versicherungsbeitrags auf der einen und der staatlichen Gewährleistung auf der anderen Seite - im Sinne eines synallagmatischen Verhältnisses nach Art. 487 des polnischen Zivilgesetzbuches. Dies bedeutet, dass auch eine eventuelle Prüfung auf ihre Äquivalenz hin unterbleiben muss, weil mangels eines Gegenseitigkeitsverhältnisses (do ut des) auch kein Vergleich von Leistungen angestellt werden kann. ${ }^{9}$

Vor dem Hintergrund, dass Beiträge und Leistungen im System der Gesundheitsfürsorge dieses Grundprinzip durchbrechen, ist auf die Aussage von Sadurski hinzuweisen, der in Anlehnung an die aristotelische Unterscheidung zwischen Verteilungs- und ausgleichender Gerechtigkeit anmerkte: „Ausgleichende Gerechtigkeit verlangt eine Gleichwertigkeit der ausgetauschten Güter und Dienstleistungen, jedoch kann die Feststellung, welche Güter und Dienstleistungen den gleichen Wert besitzen, nicht ohne vorherige Einschätzungen verteilender Natur, d.h. unter Berücksichtigung der relativen Lage der am Austausch teilnehmenden Parteien im gesamten Verteilungsgefüge, erfolgen. [...] Die austauschende Gerechtigkeit erweist sich [...] als abhängig vom Urteil der Verteilungsgerechtigkeit, denn die Gleichwertigkeit der ausgetauschten Güter [...] entpuppt sich als nichts anderes, denn ihre proportionale Verteilung nach einem vorweg angenommenen Kriterium“. ${ }^{10}$. Mit anderen Worten erweist sich die Behauptung, dass der Grundsatz der sozialen Solidarität die fehlende Äquivalenz von Beiträgen und Leistungen legitimiere, als eine gedankliche Abkürzung. Der Solidaritätsgrundsatz rechtfertigt nämlich einzig eine solche Ausgestaltung des Umfangs der gewährten Gesundheitsfürsorge, bei der die Beitragshöhe auf das persönliche Einkommen zurückzuführen ist, während der Umfang der gewährten Leistungen allein von der festgestellten Bedürftigkeit abhängt. Eine entsprechende Ungleichbehandlung verschiedener Personen ist unter dem Gesichtspunkt der Verteilungsgerechtigkeit gerechtfertigt. Deshalb sind die Pflicht des Versicherten und die gesetzliche Gewährleistung in dem Sinne gleichwertig, dass jedem Berechtigten ohne

8 Vgl. Lach, Nabycie prawa do świadczeń opieki zdrowotnej, Praca i Zabezpieczenie Społeczne 2010, Heft 11, S. 2-9.

9 Vgl. umfassender Lach, Zasada równego dostępu do świadczeń opieki zdrowotnej, Warschau 2011, S. 319 f.

10 Sadurski, Teoria sprawiedliwości. Podstawowe zagadnienia, Warschau 1988, S. $71 \mathrm{f}$. 
Rücksicht auf die individuelle Belastung des Einzelnen durch die Beitragspflicht (bzw. die Befreiung von ebendieser) derselbe Umfang und Standard von Leistungen angeboten wird.

Die Rolle und Bedeutung des Grundsatzes der sozialen Solidarität für den Aufbau des Systems der Gesundheitsfürsorge vor Augen lohnt ein Rückblick auf - einem ausführlicheren Beitrag über die Begründung der unterschiedlichen Ausgestaltung der rechtlichen Lage von (einzelnen Gruppen von) Versicherten entnommenen ${ }^{11}$ - Aussagen zum Inhalt dieses Prinzips und seiner Tragweite bei der Schaffung und Bewertung von rechtlichen Maßnahmen im Bereich des Aufbaus, der Organisation und der Finanzierung des Systems der Gesundheitsfürsorge.

\section{Der Grundsatz der sozialen Solidarität in der Literatur und Rechtsprechung}

\section{Die philosophischen Grundlagen des Solidaritätsgedankens}

Bei der Definition des Grundsatzes der sozialen Solidarität lohnt zunächst ein Blick auf die Solidarität als eine Idee, die zur letzten Jahrhundertwende Einzug in die Sozialwissenschaften hielt. Ihr Ausgangspunkt war die Vision von einer Gesellschaft als ein selbstständiges, gerade auf der Solidarität seiner Mitglieder gegründetes Wesen. Durkheim empfand die soziale Solidarität als moralische Erscheinung, die sich nach außen hin durch Recht manifestierte. ${ }^{12}$ In Anlehnung an Durkheims Gedanken betonte Duguit, dass die Solidarität als soziale Interdependenz aus zwei Teilen bestehe: Der Deckungsgleichheit menschlicher Bedürfnisse von Mitgliedern derselben sozialen Gruppe einerseits sowie der Unterscheidung ihrer Bedürfnisse und Fähigkeiten andererseits. Im Zusammenhang mit diesen Bestandteilen lässt sich auch von einer „Solidarität durch Affinität“ (Men-

11 Lach (Fn. 8), S. 111-124.

12 Für eine umfassendere Darstellung der Ideen von Durkheim vgl. Olszewski, Historia doktryn politycznych i prawnych, Warschau 1982, S. 327; aber auch: Oniszczuk, Filozofia i teoria prawa, Warschau 2008, S. 910 f. Vgl. darüber hinaus: Mik, Solidarność w prawie Unii Europejskiej. Podstawowe problemy teoretyczne, in: Mik (Hrsg.), Solidarność jako zasada działania Unii Europejskiej, Thorn 2009, S. 33 f. und Czarnota, Prawo a współczesne odmiany solidaryzmu społecznego, in: Labno (Hrsg.), Idea solidaryzmu we współczesnej filozofii prawa i polityki, Warschau 2012, S. $60 \mathrm{ff}$. 
schen tun sich zusammen, um gemeinsame Bedürfnisse zu erfüllen) oder einer „Solidarität durch Arbeitsteilung“ (Menschen tun sich zusammen, um füreinander Dienstleistungen zu erfüllen und dabei gleichzeitig verschiedenartige Bedürfnisse zu erfüllen) sprechen. Duguit erblickte die Rechtfertigung für das Bestehen des Rechtsstaates gerade in der letztgenannten ,Solidarität durch Arbeitsteilung“. ${ }^{13}$ Die „Solidarität durch Affinität" und die Gründung von Bedarfsgemeinschaften dort, wo sich Menschen mit der Erfüllung von Bedürfnissen nicht anders zu helfen wissen, sind hingegen als das Fundament und die Begründung der sozialen Absicherung im Wege einer versicherungstechnischen und treuhänderischen Lösung mitsamt dem tragenden Konzept der (zwingenden) Risikogemeinschaft anzusehen. ${ }^{14}$

Im 20. Jahrhundert entstand als Reaktion auf totalitäre Ideologien und den Zweiten Weltkrieg das Konzept des integralen Humanismus, der mit der Hervorhebung der Menschenwürde im gesellschaftlichen Leben und ihrem Vorrang gegenüber der Gesellschaft den Grundstein für die Lehre vom sozialen Personalismus legte. ${ }^{15}$ Dies bildete den Ausgangspunkt für die Beschlüsse des II. Vatikanischen Konzils und der begleitenden Enzykliken, in denen ein Vorrang privater Initiative betont wurde, während staatliche Eingriffe - entsprechend dem Subsidiaritätsprinzip - einzig zum Zwecke der Befürwortung, Förderung, Koordinierung, Unterstützung und Ergänzung erwünscht seien - so z.B. die Enzyklika Mater et magistra (1961). Der Begriff der Solidarität hielt schließlich in späteren Enzykliken Einzug. Die Enzyklika Laborem exercens (1981) sprach die Solidarität in ihrer defensiven Bedeutung an, in Bezug auf den Konflikt zwischen Arbeit und Kapital. Sie wurde darin als eine gemeinsame billige Reaktion auf die Degradierung des Menschen zum Arbeitssubjekt umschrieben. Ein abweichender Solidaritätsbegriff wurde in der Enzyklika Sollicitudo rei socialis (1987) formuliert. Dort beschrieb man Solidarität als ,einen festen und be-

13 Vgl. Duguit, Kierunki rozwoju prawa cywilnego na początku XIX wieku, in: Czarnota/Justyński (Hrsg.), Wybór tekstów źródłowych z historii doktryn politycznoprawnych, B. 2, Thorn 1988, S. 196, aber auch: Mik, ebenda, S. 34 und Olszewski, ebenda, S. $422 \mathrm{ff}$.

14 Anders stellt sich die Situation in der Grundversorgung dar, bei der die Bürgergemeinschaft alle Bürger (Bewohner) umfasst und die Zuständigkeit für die Gewährleistung der sozialen Sicherheit (wenn auch im eingeschränkten Umfang) bei der öffentlichen Hand liegt.

15 Vgl. Auleytner, Polityka społeczna, czyli ujarzmianie chaosu socjalnego, Warschau 2002, S. $198 \mathrm{f}$. 
ständigen Willen, sich für das Gemeinwohl, also das Wohlbefinden aller und eines jeden, einzusetzen“, und schrieb ihr, anders als in der Enzyklika Laborem exercens, eine positive Bedeutung als ,Engagement“ $\mathrm{zu} .{ }^{16}$

Nicht ohne Bedeutung ist dabei, dass die Hervorhebung der Würde einer Person im gesellschaftlichen Zusammenleben, ähnlich wie der Vorrang der Würde des Einzelnen vor der Gesellschaft, nicht zwangsläufig in einem Spannungsverhältnis zum, mit den Ideen von Gerechtigkeit und Solidarität verwandten, Grundsatz „Vorrang des Gemeinwohls“ stehen müssen. Der soziale Personalismus betont nämlich die Würde und Bedeutung eines Einzelnen gegenüber totalitärer Unterdrückung. In einer demokratischen Grundordnung hingegen sollte der Individualismus nicht in Egoismus umschlagen. Daraus lässt sich schließen, dass eine Grundvoraussetzung für die Entstehung einer Solidargemeinschaft (z.B. einer Risikogemeinschaft) in dem Bewusstsein zu erblicken ist, welche Ziele mit ihr verfolgt werden und welche Bedeutung sie für ihre Mitglieder hat. Dies gilt sogar dann, wenn eine Zwangsmitgliedschaft besteht. Ein auf diese Art und Weise begriffenes Bewusstsein sollte überdies den Einzelnen von Missbrauchsversuchen abhalten und die Mitglieder der Gemeinschaft zur Befolgung gewisser Regeln (Erfüllung von aus der Solidarität resultierenden Pflichten) wie zur Anerkennung der vorrangigen Stellung des Allgemeininteresses (des Gemeinwohlgrundsatzes) bewegen.

\section{Aussagen in der polnischen Lehre}

Von den kanonischen Studien der polnischen Lehre im Bereich der sozialen Absicherung ist an erster Stelle die Feststellung von Szubert zu nennen, der aufzeigte, dass ,zu den Alleinstellungsmerkmalen der Sozialversicherung die Verteilung der Beitragslast auf eine weite Gesamtheit von Verpflichteten gehört“. In diesem Kontext gebrauchte er jedoch nie den Begriff „Grundsatz der sozialen Solidarität“. ${ }^{17}$ Der Verfassungsgerichtshof stellte in Anlehnung daran in seiner Entscheidung vom 11. Februar 1992 lakonisch fest, dass der Grundsatz der sozialen Solidarität, der die Verteilung der Beitragslasten auf eine weite Gesamtheit von Sozialversi-

16 Ebenda, S. 198-203.

17 Szubert, Ubezpieczenie społeczne. Zarys systemu, Warschau 1987, S. 62 f. 
cherten gebietet, eine Rechtfertigung für die umverteilende Funktion von Versicherungen darstellt. ${ }^{18}$

Zieliński hingegen behandelte die Problematik der Solidarität im $\mathrm{Zu}-$ sammenhang mit dem Subsidiaritätsgrundsatz und dem Personalismus, welche als Bestandteile der katholischen und evangelischen Sozialphilosophie seiner Ansicht nach die ideelle Grundlage für den Sozialstaat bilden. Demnach stellt der Solidaritätsgrundsatz im Hinblick auf Sozialversicherungen eine ,mithilfe eines von allen Versicherten gemeinsam getragenen Fonds erfolgte Konkretisierung der Idee der sozialen Selbsthilfe bei der Unterstützung bedürftiger Personen“ dar. ${ }^{19}$ Zieliński berief sich darüber hinaus auf den Grundsatz der Solidarität unter Versicherten, die er mit der Gegenseitigkeitsidee gleichsetzt: Die Versicherungslast wird gemäß der Formel „einer für alle, alle für einen“ durch die Gemeinschaft der Versicherten getragen. Dabei darf der Grundsatz der Gegenseitigkeit (der Solidarität unter Versicherten) aber nicht mit dem schuldrechtlichen Gegenseitigkeitsprinzip (Leistungssynallagma) verwechselt werden, da dieses den Sozialversicherungen fremd ist. ${ }^{20}$

Auch Piotrowski betont die Bedeutung des Solidaritätsgrundsatzes für die Risikogemeinschaft, in der es zu einer Verteilung der Verantwortung unter allen Versicherten kommt. Er stellt fest, dass das Wesen der Risikogesellschaft in dem Bedürfnis besteht, die durch Zufallsereignisse entstandenen Vermögensbedürfnisse auf eine Vielzahl von Personen zu verteilen, die auf ähnliche Weise von einem nicht absehbaren Risiko betroffen sind. ${ }^{21}$

Eine ähnliche Ansicht vertritt auch Jończyk, der darauf hinweist, dass in der Literatur ,in Bezug auf Risikogemeinschaften im rechtlichen Sinne selten die Rede von Solidarität ist, obwohl diese jeglichen Formen der sozialen Absicherung zugrunde liegt".22 Besonders hervorzuheben ist folgende Aussage dieses Autors: „Die Solidaritätsthematik betrifft Pflichten, Lasten, individuelle Beiträge, gemeinsame Anstrengungen, nicht hingegen die Verteilung von Geld- und Sachleistungen“, denn „die Solidarität einer

18 VerfGH v. 11.02.1992, K 14/91, OTK 1992, H. 1, Pos. 7.

19 Zieliński, Ubezpieczenia społeczne pracowników. Zarys systemu prawnego część ogólna, Warschau - Krakau 1994, S. 20.

20 Vgl. ebenda, S. $17 \mathrm{ff}$. sowie $130 \mathrm{f}$.

21 Vgl. Piotrowski, Zabezpieczenie społeczne. Problematyka i metody, Warschau 1966, S. 173.

22 Jończyk (Fn. 3), S. 38. 
Risikogemeinschaft drückt sich durch die Finanzierung (mittels individueller Beiträge) der sozialen Absicherung aus und sollte nicht mit dem Ausgleich von finanziellen Lasten im Rahmen von Risiko- bzw. Solidaritätsgemeinschaften verwechselt werden". ${ }^{23}$ Aus diesem Grund merkte Jończyk an, dass ,im Kontext der Aufteilung von Leistungen vielmehr von Gerechtigkeit als von Solidarität die Rede ist".${ }^{24}$ In Bezug auf den Inhalt des Solidaritätsgrundsatzes stellte der Autor fest, die Schaffung einer Risikogemeinschaft (auch in Bezug auf das Risiko, nicht gesund zu sein) beruhe auf der Prämisse, es seien von ihr Rechtsträger umfasst, die - wenngleich in verschiedenem Maße - imstande und bereit sind, die Kosten des Schutzes gegen die Folgen des Eintretens eines bestimmten Risikos zu tragen. Gerade im Hinblick auf Systeme sozialer Sicherheit insgesamt und Systeme der Gesundheitsfürsorge im Besonderen beziehen sich die genannten Unterschiede nicht lediglich auf die Fähigkeit, das System mitzufinanzieren, sondern genauso auf die „Risikoanfälligkeit“ i.S. der individuellen Wahrscheinlichkeit des Eintretens eines Zufallsereignisses, welche als Grundlage für den rechtlichen Begriff des sozialen Risikos dient. Ebenso wenig besteht ein ,symmetrisches Verhältnis zwischen dem eigenen Beitrag und den empfangenen Leistungen“, vielmehr spiegelt diese Asymmetrie das wahre Wesen der Solidarität wider: „Obgleich sich alle am System beteiligen, wenn auch oftmals in ungleichem Maße sowie in längeren oder kürzeren Mitgliedszeiträumen, so empfangen doch einzig die Personen eine Entschädigung, die tatsächlich einen Schaden erlitten haben“. ${ }^{25}$ Aus diesem Grund - und ferner zur Verhinderung von „Egoismus und Spekulation“ - muss die Schaffung einer Risikogemeinschaft obligatorisch sein, da diese eine „Garantie für die Solidarität und Tragfähigkeit der Sozialversicherungen“ darstellt. ${ }^{26}$ Unmittelbar zum System der Gesundheitsfürsorge sprach sich Jończyk wie folgt aus: „Im Vergleich mit der Zeit der Volksrepublik Polen ist es sogar zu einer Vertiefung des Solidaritätsgrundsatzes gekommen, was sich beispielsweise in einer deutlich größeren Finanzierungsvielfalt im Bereich der Gesundheitsrisiken zeigt [...]. Angesichts der auseinandergehenden Einkommensschere in der Bevölkerung (die ihren Hauptverdienst mit Arbeitslöhnen bestreitet) ist zwangsläufig eine wachsende Diskrepanz zwischen Beitragszahlungen

23 Ebenda.

24 Ebenda.

25 Ebenda.

26 Ebenda, S. 39. 
und empfangenen Leistungen zu erwarten“. ${ }^{27}$ Dies verleitete den Autor zu einer klaren Stellungnahme: „Es handelt sich somit um eine gesetzlich auferlegte Solidarität, die im Lichte der Staatsorganisation der III. Republik keine hinreichende ethische, soziale und wirtschaftliche Rechtfertigung findet" ${ }^{\text {. }}{ }^{28}$

\section{Der Solidaritätsgrundsatz und sozialer Solidarismus in der Rechtsprechung des Verfassungsgerichtshofs und des Obersten Gerichts}

An dieser Stelle soll nun die Rechtsprechung des Verfassungsgerichtshofs zum Solidaritätsgrundsatz näher beleuchtet werden. Im bereits zitierten Urteil vom 11. Februar $1992^{29}$ befand der Gerichtshof, dass ,eine Begründung [...] für die Umverteilungsfunktion der Sozialversicherungen im Grundsatz der sozialen Solidarität liegt, der eine Verteilung der Beitragslast auf die Allgemeinheit der Versicherungspflichtigen verlangt". Ferner hob der Verfassungsgerichtshof im Urteil vom 7. Januar 200430, Az. K 14/03 ${ }^{31}$, den finanziellen Aspekt des Solidaritätsgrundsatzes hervor und stellte fest: „Der Zugang zu öffentlich finanzierten Leistungen muss für alle Bürger unabhängig von ihrer finanziellen Lage gleichermaßen möglich sein. Die von der untersuchten Vorschrift normierte Gleichheit beim Zugang zu Leistungen der Gesundheitsfürsorge stellt eine Fortbildung des allgemeinen Gleichheitssatzes aus Art. 32 der Verfassung sowie dem Konzept der sozialen Solidarität dar. Die Voraussetzungen für die Nutzung der Leistungen der Gesundheitsfürsorge richten sich nämlich unabhängig von der Beteiligung des einzelnen Bürgers nach der Aufbringung der öffentlichen Mittel zur Finanzierung dieser Leistungen“.

Ähnlich formulierte das Oberste Gericht den Solidaritätsgrundsatz im Urteil vom 9. Juni $2005^{32}$, indem es feststellte, dass „die Organe der öf-

27 Ebenda, S. 309.

28 Ebenda. Vgl. auch: Jończyk, Transformacja ubezpieczeń społecznych i ochrony zdrowia, in: Szurgacz (Hrsg.), Konstytucyjne problemy prawa pracy i zabezpieczenia społecznego. Referaty na XV Zjazd Katedr i Zakładów Prawa Pracy i Ubezpieczeń Społecznych, Wrocław 1-2 czerwca 2005 r., Breslau 2005, S. 120 f.

29 VerfGH v. 11.02.1992, K 14/91, OTK 1992, H. 1, Pos. 7.

30 VerfGH v. 07.02.2004, K14/03.

31 Poln. GBl. 2004, Nr. 5, Pos. 37.

32 Oberstes Gericht v. 09.06.2005, III CK 626/04 = OSNC 2006, Nr. 5, Pos. 89. 
fentlichen Gewalt zur Erhaltung eines öffentlich finanzierten Systems der Gesundheitsfürsorge verpflichtet worden sind, das den Grundsätzen der allgemeinen Zugangsgleichheit und der Solidarität in Gestalt der Verwendung der gleichen medizinisch angemessenen Verfahren gegenüber Bedürftigen ungeachtet deren Beteiligung an der Gestaltung der Einnahmen des Systems Folge leistet".

Vor diesem Hintergrund ist anzumerken, dass Untersuchungen der Rechtsprechungspraxis im Schrifttum ergaben, dass die „Termini ,Solidarität' $[\ldots]$ und ,Solidarismus' synonym verwendet werden und die jeweilige Begriffswahl mehr auf stilistischen denn auf sachlichen Überlegungen beruht“". ${ }^{33}$ In der Literatur wurde sogar die Ansicht vertreten, dass „oftmals der geltende Sprachgebrauch über die Unterscheidung zwischen den Begriffen ,Solidarität' und ,Solidarismus' und ihre Austauschbarkeit bestimmt. Vom sprachlichen Brauchtum hängt demzufolge unsere Entscheidung $\mathrm{ab}$, wie wir uns bei der Anwendung der Begriffe Solidarismus und Solidarität entscheiden und ob von einem solidaristischen oder solidarischen Staat in Rede ist. Unter diesen Umständen sind jegliche Versuche, die genauen Unterschiede zwischen den beiden Begriffen herzauszuarbeiten, zum Scheitern verurteilt. Deshalb bestehen auch keine Hindernisse, beide Begriffe synonym zu verwenden“. ${ }^{34}$ Dieser Annahme kann kaum widersprochen werden.

Das Problem der korrekten Terminologie bei der Behandlung der theoretischen Grundlagen und des Aufbaus von Techniken zur Verwirklichung der Idee von einer sozialen Absicherung wurde bereits früher in Bezug auf die Rechtsprechung des Obersten Gerichts diskutiert. ${ }^{35}$ Zwischen dem Grundsatz der sozialen Solidarität und dem sozialen Solidarismus besteht ein wesentlicher Unterschied. Gemäß der Definition im „Wörterbuch der polnischen Sprache" bezeichnet man als Solidarismus eine Mitte des 19. Jh. entstandene sozialpolitische Denkrichtung, die von einer natürlichen Interessengemeinschaft ausgeht, welche sich aus verschiedenen Gruppen

33 Vgl. Bielska-Brodziak/Bogucka, Solidarność jako termin prawny i jego funkcjonowanie w praktyce orzeczniczej, in: Labno (Hrsg.), Idea solidaryzmu we współczesnej filozofii prawa i polityki, Warschau 2012, S. $208 \mathrm{ff}$.

34 Vertiefend zu den Termini „Solidarismus” und „Solidarität” vgl. Tkacz/Wentkowska, O naturze teoretyczno-prawnej i uwikłaniach pojęcia „solidaryzm”, in: Labno (Hrsg.), Idea solidaryzmu we współczesnej filozofii prawa i polityki, Warschau 2012, S. $107 \mathrm{ff}$.

35 Vgl. Lach, Glosse zum Beschluss des Obersten Gerichts v. 27. 04.2005, II UZP 2/05, GSP Prz. Orz. 2006, Nr. 2, S. 163 ff. 
von Bürgern im Staat unabhängig von finanziellen und sozialen Unterschieden zusammensetzt. Der Grundsatz der sozialen Solidarität betrifft hingegen allein die Mitglieder der Gemeinschaft aller Versicherten - einer Risikogemeinschaft - und nicht die Gesamtheit der Bürger (woran auch die allgemeine Natur der „Gesundheitsversicherung“ nichts zu ändern vermag). Jończyk führte dazu nüchtern an: „Die Solidarität einer Risikogemeinschaft ist von einem Gruppencharakter geprägt [...]. Es handelt sich bei ihr um keine gesamtmenschliche, globale [...], nationale Solidarität.“36

Der zutreffenden Bemerkung von Ślebzak ${ }^{37}$ über das Sozialversicherungswesen, dass in einer Gemeinschaft von Solidarität sowohl in den Beziehungen zwischen den Versicherten untereinander, als auch zwischen ihnen und den Leistungsempfängern ${ }^{38}$, den Versicherten und den Beitragszahlern (im Kontext der Zuweisung von Beiträgen), den Leistungsempfängern untereinander und, schließlich, den Leistungsempfängern und dem Staat ausgegangen werden könne, ist anzufügen, dass in Bezug auf die „Gesundheitsversicherung“ einzig von der Solidarität zwischen Versicherten gesprochen werden kann. Dies geht mit der Tatsache einher, dass nur sie alleine die Risikogemeinschaft bilden. Patienten, die Leistungen empfangen, gehören ihr insofern an, als dass sie auch nach Beginn der Durchführung einer konkreten Leistung weiterhin in den Genuss der gesetzlichen Garantie kommen. Angesichts der Belastung durch die Beitragspflicht - die nebenbei bemerkt als mit der Einkommensteuer strikt verbundene, einzig Versicherungspflichtigen, in der Praxis vor allem Erwerbstätigen auferlegte quasi-Zwecksteuer zur Erhaltung der Gesundheitsfürsorge angesehen werden kann - sowie der Finanzautonomie der Gesundheitsfürsorge spielt die Solidarität im Falle der "Gesundheitsversicherung“ in den anderen, oben genannten Verhältnissen keine Rolle. Dies ist darauf zurückzuführen, dass im Rahmen dieses Systems eine als ,Zwischenpersönlicher Ausgleichstransfer" bezeichnete Umverteilung stattfindet. Leistungen zugunsten der einen werden danach aus Beiträgen der anderen finanziert und mithilfe des Grundsatzes der sozialen Solidarität gerechtfertigt. Eine

36 Jończyk, Ubezpieczenie zdrowotne, Praca i Zabezpieczenie Społeczne 2003, S. 4.

37 Vgl. Ślebzak, Zasada solidarności w ubezpieczeniu emerytalnym, in: Kubot/ Kuczyński (Hrsg.), Z zagadnień prawa pracy i prawa socjalnego. Księga Jubileuszowa z okazji 70-tych urodzin Profesora Herberta Szurgacza, Breslau 2011, S. 540 .

38 Unter Berücksichtigung der gesetzlichen Terminologie (Art. 2 LÖffGG) ist von Patienten, die Leistungen in Anspruch nehmen, die Rede. 
andere, umfassendere, auf die nationale oder staatsbürgerliche Gemeinschaft abstellende Auslegung des Grundsatzes der sozialen Solidarität, findet keine Legitimation im Aufbau des Systems der Gesundheitsfürsorge.

In Bezug auf die axiologischen Grundlagen der Sozialversicherung und die einzelnen Techniken zur Verwirklichung dieser Idee sollte auf den Begriff der sozialen Solidarität i.S. einer vorausgesetzten Verbundenheit der Mitglieder in Zwangsverbänden zur gegenseitigen Absicherung von Leistungsansprüchen im Falle des Eintritts von sozialen Risiken zurückgegriffen werden. Dieser Grundsatz besitzt einen eigenen, bestimmten Inhalt (vgl. III.) und kann in keiner Weise mit der politischen Doktrin des Solidarismus gleichgesetzt werden, die sich auf eine weitere Gemeinschaft sowie sozialpolitische Aspekte bezieht.

\section{Aussagen aus der deutschen Doktrin}

Über das Sozialrecht und seine axiologischen Grundlagen wurden in der deutschen Rechtslehre zahlreiche Aussagen gemacht. Die Problematik des in Deutschland als Solidarprinzip bezeichneten Erfassungsgrundsatzes behandelt unter anderem Volkmann ${ }^{39}$, der vor allem die kooperative, auf die Verhältnisse zwischen Rechtsubjekten bezogene Dimension der Solidarität betont. Diese tritt vor allem in den Pflichten zur Berücksichtigung des Entfaltungsspielraums des Gegenübers, der Kooperations- und der Einstandspflicht ${ }^{40}$ in Erscheinung und konstatiert eine Verantwortung der Rechtsträger. Der Autor stellt deshalb die These der Solidarität als Grundpflicht auf. ${ }^{41}$ Über den Sozialstaat schrieb Volkmann: „Der Sozialstaat erscheint im Lichte der Solidarität nicht mehr als bürokratisch-institutioneller Komplex, mit dem eine mit wichtigeren Dingen beschäftigte Gesellschaft ihre Sozialfälle entsorgt und ihre hilfsbedürftigen Mitglieder zu passiven Klienten zurichtet, sondern als Derivat einer sämtliche Mitglieder umspannenden Verantwortungsgemeinschaft" ${ }^{42}$ Als Ausfluss der Solidarität werden demnach nicht nur soziale Rechte verstärkt, sondern auch das Gefühl, dass jeder Bürger ein „Solidaritätsschuldner“ ist, da „der Sozial-

39 Vgl. Volkmann, Solidarität - Programm und Prinzip der Verfassung, Tübingen 1998.

40 Der Pflicht zur Beteiligung an den Kosten der Solidargemeinschaft.

41 Vgl. Volkmann (Fn. 39), S. 378 f.

42 Ebenda, S. 395. 
staat selbst nur das Mittel ist, dessen sich die Bürger bedienen, um ihre Solidarität zu organisieren". ${ }^{43}$ Volkmann stellt fest, dass das Solidarprinzip in diesem Zusammenhang eine Legitimation zum Eingriff in die Rechtslage des Einzelnen und zur Auferlegung sowie Differenzierung von Lasten wie z.B. die Verknüpfung der Beitragshöhe mit dem persönlichen Einkommen liefert. ${ }^{44}$

Becker ${ }^{45}$ bemerkte zutreffend, dass der Begriff „Solidarität“" zunächst nur unspezifisch das Füreinander-Einstehen-Müssen bezeichnet und als Rechtsbegriff der gesetzlichen Ausformung bedarf. Durch diese werden aus möglichen moralischen Verpflichtungen vor allem konkrete Zahlungsverpflichtungen. Weiterhin schrieb er: „Solidarität wird im Sozialrecht weniger durch die Schaffung von Leistungssystemen selbst als vielmehr durch die Anordnung eines finanziellen Ausgleichs innerhalb von Systemen hergestellt. Insofern spielt es eine wesentliche Rolle, welche Personen in welche Systeme einbezogen und wie die zu erbringenden Sozialleistungen finanziert werden." Bezugnehmend auf das Prinzip der Eigenverantwortung als Kehrseite der Solidarität betonte Becker den kollektiven Charakter des letztgenannten Grundsatzes.

Ferner kann auf die Arbeit von Ruland mit einer nahezu schlagwortartigen Darstellung des Solidaritätsbegriffs, mitunter in Bezug auf die Vorschriften des Sozialversicherungsrechts, hingewiesen werden. Der Autor merkte vor allem an, dass Solidarität gleichbedeutend mit einer „Entindividualisierung“ von Risiken ist ${ }^{46}$, was jedoch nicht auf einen Verzicht auf die Eigenverantwortlichkeit von Leistungsempfängern im Rahmen solidarischer Systeme hinauslaufen kann, da Solidarität gleichzeitig Pflichten mit sich bringt wie die Loyalitätspflicht gegenüber der Risikogemeinschaft. Aus diesem Grund verletzt seine Pflichten, wer ohne einen entsprechenden Bedarf auf Leistungen der Gesundheitsfürsorge zurückgreift bzw.

43 Ebenda, S. 396. Auch andere Autoren haben angemerkt, dass das Sozialstaatsprinzip ebenfalls vom Gesichtspunkt der Belastung von Bürgern, der sich auch als Solidarprinzip bezeichnen lässt, erfasst werden kann. Vgl. Bley/Kreikebohm/Marschner, Sozialrecht, Neuwied 2007, S. 27.

44 Vgl. Volkmann (Fn. 39), S. 400.

45 Vgl. Becker, Sozialrecht und Sozialrechtswissenschaft, ZÖR 2010, S. 616 f.

46 Zacher schreibt in diesem Zusammenhang von der Kollektivierung individueller Risiken. Vgl. Zacher, Individuelle und soziale Sicherung gegen die Notfälle des Lebens in der sozialen Marktwirtschaft, Berlin 1973, S. 142. 
selbst ein Ereignis schuldhaft herbeiführt, das Leistungsansprüche auslöst. ${ }^{47}$ Dabei ist der Anmerkung von Gitter beizupflichten, dass ,ohne Eigenverantwortung Solidarität anonym und missbräuchlich“ wird, „Eigenverantwortung ohne Solidarität egoistisch ist" ${ }^{48}$

Das Solidarprinzip als ,axiologische Grundlage des Sozialrechts“ bezeichnend $^{49}$, trug Faude hingegen vor, dass es sich um ein Konzept gemeinsamer Verantwortung für das Schicksal eines jeden Einzelnen im Gegensatz zum individualistischen Konzept des Privatrechts handele. Danach sei sie auf verschiedenen Organisationsebenen realisierbar, woraufhin er die staatliche Ebene mit Beispielen wie Sozialhilfe, Unterstützungsleistungen und soziale Entschädigung sowie die Ebene der einzelnen Systeme sozialer Absicherung (darunter auch der Gesundheitsfürsorge) hervorhob. Ähnlich wie Ruland, wies auch Faude auf die aus dem Solidarprinzip abgeleitete Notwendigkeit des ,solidarischen Verhaltens“ hin und stellte die verschiedenen Dimensionen ihrer Bewertung vor: 1) das Verhalten des Rechtsträgers (böswilliges Verhalten verdient keine Solidarität), 2) den Zweck, aus dem die solidarische Risikogemeinschaft entstanden ist (z.B. die Einschränkung der Risikodefinition auf typische Folgen und Ursachen eines gegebenen Zufallsereignisses durch den Ausschluss einer Garantie in Fällen einer Eigenverschuldung bei seiner Herbeiführung) und 3) garantierte Leistungen (bewertet wird die Art und Weise der Inanspruchnahme der Leistung sowie die Kooperation des Leistungsempfängers). ${ }^{50}$

Im deutschen Schrifttum wird das Solidarprinzip zumeist im Verhältnis mit den Grundsätzen der Gegenseitigkeit und Gleichwertigkeit von gezahlten Beiträgen und empfangenen Leistungen angeführt, die zwar die Grundlage für Versicherungen im Privatrecht bilden, in der „Gesundheitsversicherung" aber - wie bereits festgestellt - grundsätzlich ausgeschlos-

47 Vgl. Ruland, Solidarität, NJW 2002, S. 3518 f.; aber auch: Borchert, Die Rolle der Patienten und Versicherten im neustrukturierten GKV-System, in: Heinze/Schmitt (Hrsg.), Festschrift für Wolfgang Gitter zum 65. Geburtstag am 30. Mai 1995, Wiesbaden 1995, S. 133-144.

48 Gitter, Strukturen der Reform der gesetzlichen Krankenversicherung, Die soziale Gerichtsbarkeit 1991, S. 86.

49 So ähnlich: Heinig, Der Sozialstaat im Dienst der Freiheit, Tübingen 2008, S. 121-133.

50 Vgl. Faude, Selbstverantwortung und Solidarverantwortung im Sozialrecht. Strukturen und Funktionen der sozialrechtlichen Relevanz des Selbstverschuldens des Leitungsberechtigten, Bonn 1983, S. 33 f. und 36 ff. 
sen sind. In diesem Zusammenhang kann auf die Meinung von Kirchhof ${ }^{51}$ verwiesen werden, der bei der Ermittlung des Inhalts des Solidarprinzips auf vier grundlegende, eine äquivalente Gestaltung des Verhältnisses von Beitragszahlungspflicht und Leistungsberechtigung aus einer Sozialversicherung ausschließende Formen der Umverteilung abstellte. Erstens - den intertemporalen Ausgleich, bei dem es zu einer zeitlichen Verschiebung zwischen der Entrichtung von Beiträgen und dem Erhalt von Leistungen kommt, wobei diese Verschiebung weiterhin denselben Rechtsträger betrifft (beispielsweise im Falle von kapitalanlagenfinanzierten Rentenversicherungen), zweitens - den generationenübergreifenden Ausgleich (z.B. durch Umverteilung finanzierte Renten), und drittens - den institutionsübergreifenden Ausgleich in Bezug auf Verschiebungen zwischen den Haushalten unterschiedlicher Institutionen der sozialen Absicherung (beispielsweise den bei einer Mehrzahl von Gesundheitsversicherungsträgern notwendige Mechanismus zur Vorbeugung der Risikowahl). ${ }^{52}$ Am bedeutendsten für die Funktionsfähigkeit eines Systems der Gesundheitsfürsorge erscheint jedoch - wie bereits erwähnt - die vierte Form der Umverteilung, der sog. personenübergreifende Ausgleich, nach welchem die Leistungen des einen durch Beiträge der anderen finanziert werden. ${ }^{53}$ Kirchhof führte des Weiteren aus, dass die vom Solidarprinzip legitimierte Umverteilung nachreguliert werden kann, und zwar durch Vorschriften über Beiträge (im Wege der Einforderung der finanziellen Mitverantwortung von anderen Trägern als den Versicherten, etwa den Versicherern oder dem Staat - durch Zuschüsse) sowie Leistungen (die auch anderen Trägern als den Versicherten, wie etwa ihren Familienmitgliedern, zugesprochen werden bzw. aus gesetzlich auferlegten außerversicherungsrechtlichen Aufgaben der öffentlichen Hand resultieren können, wie z.B. die Mitversicherung von Arbeitslosen, Empfängern von Sozialhilfe usw.). ${ }^{54}$ Im Ergebnis einer Untersuchung der deutschen Sozialversicherungssysteme stellte Kirchhof fest, dass soziale Elemente gerade in der Gesundheitsversiche-

51 Vgl. Kirchhof, Das Solidarprinzip im Sozialversicherungsbeitrag, in: Schulin (Hrsg.), Sozialfinanzverfassung: 5. Sozialrechtslehrertagung, Wiesbaden 1992, S. $65 \mathrm{ff}$.

52 Daneben bleibt zu bedenken, dass ähnliche Mechanismen auch im Gesetz vom 6. Februar 1997 über die allgemeine Krankenversicherungspflicht vorgesehen waren. Entsprechend Art. 135 Abs. 1 beteiligten sich die Krankenkassen am finanziellen Ausgleich von einkommens- und kostenbedingten Unterschieden.

53 Vgl. Kirchhof(Fn. 51), S. 65 ff.

54 Vgl. ebenda, S. $67 \mathrm{ff}$. 
rung deutlich stärker als in anderen Bereichen zur Geltung kommen. Er betonte, die Beitragshöhe hänge stets vom Einkommen des Versicherten $\mathrm{ab}$, weshalb - trotz des Abstellens der Versicherungsgarantie auf eine Sachleistungszusage für medizinische Bedarfsfälle anstelle eines pauschalen Ausgleichs vom Verdienstausfall - eine Differenzierung der Rechtslage der Versicherten je nach ihren Vermögensverhältnissen stattfindet, was ein Indiz für die Anwendung des Solidarprinzips darstellt. ${ }^{55}$ Schließlich wies er ferner auf die Mitversicherung von Familienmitgliedern hin. ${ }^{56}$

\section{Zusammenfassung - Inhalt des Grundsatzes der sozialen Solidarität in der ,, Gesundheitsversicherung“}

Bei dem Versuch, den Grundsatz der sozialen Solidarität im „Gesundheitsversicherungswesen“" mit Blick auf die polnische Lehre und Rechtsprechung des Verfassungsgerichtshofs und des Obersten Gerichts einerseits und das deutsche Schrifttum andererseits zu definieren, muss vor allem auf die unterschiedlichen Akzente bezüglich der Bedeutung und Funktion des Solidaritätsprinzips abgestellt werden. Dies wirkt sich ohnehin auf die Bewertung der Funktionalität eines Systems und seine außerrechtliche Wahrnehmung in der Öffentlichkeit aus. In der Begründung des Urteils vom 7. Januar $2004{ }^{57}$ stellte der Verfassungsgerichtshof fest, dass aus der Solidarität „ein gleicher Zugang zu Leistungen der Gesundheitsversorgung" hervorgeht, während die Inanspruchnahme von Leistungen der Gesundheitsversorgung „unabhängig von der Beteiligung einzelner Mitglieder der Bürgergemeinschaft an der Finanzierung durch öffentliche

55 Vgl. ebenda, S. 75. Diesen Gesichtspunkt als Ausdruck von Solidarität zwischen dem einzelnen Versicherten und anderen Mitgliedern der Versichertengemeinschaft (Risikogemeinschaft) betonte auch Ost, in: Ost/Mohr/Estelmann (Hrsg.), Grundzüge des Sozialrechts, München 1998, S. 15 f.

56 Vgl. Kirchhof (Fn. 51), S. 75. Auch Haverkate sah - in Bezug auf die Gesundheits- bzw. Krankenversicherung - die unentgeltliche Mitversicherung von Familienmitgliedern als das bedeutendste Beispiel (neben der unterschiedlichen Beitragshöhe trotz identischer gesetzlicher Garantien) für die Verwirklichung des Solidarprinzips als Grundgedanken des gesamten deutschen Sozialversicherungswesens. Vgl. Haverkate, Gleichheitsprobleme an den Nahtstellen der Sozialleistungssysteme - Am Beispiel der Alterssicherung, in: Beyerlin/Bothe/Hofmann/Petersmann (Hrsg.), Recht zwischen Umbruch und Bewahrung, Völkerrecht, Europarecht, Staatsrecht, Festschrift für Rudolf Bernhardt, Berlin 1995, S. 394.

57 VerfGH v. 07.01.2004, K 14/03. 
Mittel“ möglich sein muss. Ohne diesen Thesen grundsätzlich zu widersprechen sollte angemerkt werden, dass dieses Verständnis von Solidarität vor allem den Erwerb von Leistungsberechtigungen durch weniger wohlhabende Bürger auf Kosten der Vermögenden rechtfertigt.

Analog bemerkte man im polnischen Schrifttum, dass ,der Grundsatz der sozialen Solidarität (des sozialen Solidarismus) i.S. der „Gesundheitsversicherung" auf einer spezifischen Anonymität des aus Beiträgen finanzierten Fonds beruht. Grundsätzlich haben die Beitragszahler keine Kenntnis davon, wessen Leistungen aus den von ihnen eingezahlten Mitteln finanziert werden. Ungeachtet der Höhe des von ihnen gezahlten Beitrags kommt ihnen dieselbe Leistungsberechtigung zu. Demzufolge erwirbt keiner der Versicherten größere Anrechte beim Zugang zu Versicherungsleistungen allein weil seine Beiträge höher ausfallen als die der anderen“".58

Demgegenüber wird das Solidarprinzip im deutschen Recht gänzlich anders (und zutreffender) als im polnischen Recht ausgelegt. Aus dem Solidarprinzip werden vor allem Pflichten für die Mitglieder der Versichertengemeinschaft abgeleitet: Die Zwangsteilnahme, die Pflicht zur Zahlung von Beiträgen - trotz fehlender Gegenseitigkeit von Beitragshöhe und erhaltenen Leistungen - und die Pflicht, sich solidarisch zu verhalten. Dieser für Leistungsempfänger verpflichtend wirkende und auf Verantwortlichkeit und einer bewussten Haltung beruhende Aspekt des Solidaritätsgrundsatzes verdient, so scheint es, eine deutlich stärkere Betonung sowohl in der Rechtsprechung als auch der Lehre. Seine Ausklammerung oder gar Nichtberücksichtigung führt zu einer fehlenden Identifikation der Leistungsempfängern mit dem System, was zu Versuchen führt, sich der Beitragspflicht um jeden Preis zu entziehen (u.a. durch einen Rückzug in die Grauzone oder die Auslagerung der unternehmerischen Tätigkeit ins Ausland) oder die garantierten Leistungen zu missbrauchen.

In Anspielung auf die angeführte Aussage von Jończyk sollte einmal mehr unterstrichen werden, dass ,die Thematik der Solidarität vor allem

58 Nowak-Kubiak/Łukasik, Ustawa o świadczeniach opieki zdrowotnej finansowanych ze środków publicznych, Kommentar zu Art.65, Warschau 2006, S. 126-128. Am Rande sei angemerkt, dass mit Ausnahme der kapitalanlagenfinanzierten Alterssicherung jeder andere Versicherungsfonds in dem Sinne anonym bleibt, als dass ein Beitrag (seine Höhe) nicht an bestimmte versicherte Personen gebunden ist. Das Fehlen einer Verbindung zwischen Leistungsumfang und Beitragshöhe hingegen hat mit der Anonymität eines solchen Fonds nichts zu tun vielmehr handelt es sich dabei um die Quintessenz des Grundsatzes der sozialen Solidarität. 
Pflichten, Lasten, individuelle Beiträge und gemeinsame Anstrengungen betrifft und nicht die Verteilung von Geld- und Sachleistungen“, während „im Hinblick auf die Verteilung von Leistungen vielmehr auf Gerechtigkeit denn auf Solidarität abgestellt wird“. ${ }^{59}$ Anders ausgedrückt kann der Gerechtigkeitsgrundsatz bei der Suche nach einer axiologischen Begründung für die unterschiedliche rechtliche Behandlung von Leistungsempfängern (das Differenzierungskriterium sollte aus der angenommenen Werteordnung hervorgehen) - im Zusammenhang mit dem Grundsatz des gleichen Zugangs zu Leistungen der öffentlich finanzierten Gesundheitsversorgung - sehr wohl eine Unterscheidung bei der Gewährung von Rechtspositionen rechtfertigen, während der Grundsatz der sozialen Solidarität vielmehr die Differenzierung der Pflichten von Teilnehmern dieses Systems betrifft.

Vor dem Hintergrund der angeführten Aussagen aus dem polnischen und deutschen Schrifttum sowie unter Berücksichtigung der Rechtsprechung des Verfassungsgerichtshofs lässt sich zusammenfassend festhalten, dass die aus den gegebenen Interdependenzen hervorgehende Übernahme von gewissen Lasten und Pflichten durch eine Gemeinschaft, welche zu einem sozialen Ausgleich führen, den Inhalt des Grundsatzes der sozialen Solidarität ausmacht. Der Solidaritätsgedanke geht nämlich von einer „Vergemeinschaftung“ („Entindividualisierung“) von Risiken und nicht von einem jeweiligen individuellen Risiko oder Bedürfnis (personenübergreifender Ausgleich) aus. Die „Vergemeinschaftung“ manifestiert sich vor allem in der Zahlung von einkommensabhängigen Beiträgen. Die Solidarität unter Mitgliedern einer Gemeinschaft i.S. ihrer gegenseitigen Verbundenheit entspricht dabei einem Vorrang des Allgemeinwohls gegenüber dem Wohl des Einzelnen. Das Allgemeinwohl sowie Handlungen zu dessen Gunsten sind sowohl Ziel als auch Folge des Bestehens einer Solidargemeinschaft. Daher folgt aus dem Grundsatz der sozialen Solidarität u.a. eine Loyalitätspflicht gegenüber den anderen Mitgliedern der Gemeinschaft in Gestalt der Pflicht zur aktiven Minimierung der Wahrscheinlichkeit eines Risikoeintritts und der potentiellen Folgen einerseits sowie der angemessenen Nutzung der garantierten Leistungen und der Notwendigkeit des Zusammenwirkens und Berücksichtigung der Interessen anderer Mitglieder, etwa durch die Gewährleistung gleicher Garantien bei ungleichen Belastungen andererseits.

59 Ebenda, S. 127. 
Bei der Ermittlung einer verfassungsrechtlichen Grundlage für die gesetzlich bestimmten Lösungen zur Verwirklichung des Grundsatzes der sozialen Solidarität kann auf Art. 68 Abs. 2 der polnischen Verfassung hingewiesen werden. Der dort statuierte Grundsatz des gleichen Zugangs zu Leistungen der Gesundheitsfürsorge betrifft nämlich in erster Linie die Vermögensunterschiede der Bürger. Unabhängig von dem eigenen Beitrag (dessen Höhe nach der Versicherungsgrundlage und dem erzielten Einkommen, jedoch nicht nach der Zahl der mitversicherten Familienmitglieder oder dem individuellen Risikopotenzial von Gesundheitsbeeinträchtigungen beim Versicherten und seinen Mitversicherten berechnet wird) sollte jeder Bürger im gleichen Maße (d.h. ohne Unterscheidung in Bezug auf die Reichweite und Voraussetzungen dieser Fürsorge) auf die im Rahmen des Systems der Gesundheitsfürsorge garantierten, öffentlich finanzierten Leistungen zurückgreifen können.

Dennoch sollte einmal mehr bedacht werden, dass sich der Grundsatz der sozialen Solidarität im Wesentlichen auf die Pflichten und weniger auf die Berechtigungen der Versicherten bezieht. Anhand dieses Prinzips lassen sich die Beteiligung an der Risikogemeinschaft und der Grad ihrer Finanzierungspflicht ausmachen, während das Recht auf Leistungen auf der bloßen Mitgliedschaft im System beruht und ihr Umfang von der individuellen Bedürftigkeit abhängt. Die Teilnahme an der Risikogemeinschaft kann demnach in einem solidarisch geprägten Leistungssystem zu keinem Verzicht des Versicherten auf Eigenverantwortung führen, da Solidarität mitunter, wenn nicht vor allem, eine an Verantwortung und bewusste Eigenhaltung anknüpfende Einstandspflicht fordert - insbesondere die Pflicht zur Sorge um die eigene Gesundheit.

Nur am Rande sei angemerkt, wie problematisch sich die Frage einer eventuellen Sanktionierung von Verstößen gegen solche Pflichten darstellt. Dies liegt zum einen an den beschränkten Ermittlungsmöglichkeiten (vor allem in Bezug auf die Feststellung, ob ein Ereignis tatsächlich zufällig eingetreten ist, d.h. inwiefern sich das Eigenverhalten des Leistungsempfängers auf den Eintritt des Gesundheitsrisikos bzw. die Behandlungsdauer und -effektivität ausgewirkt hat), aber vor allem auch an den vorhandenen rechtlichen Mitteln, über die der Versicherungsträger (der Nationale Gesundheitsfonds) verfügt. Wie bereits an anderer Stelle angemerkt ${ }^{60}$, kann ein eventuelles Verschulden des Versicherten nicht dazu

$60 \operatorname{Lach}($ Fn. 8), S. 239 ff. 
führen, dass ihm der Erwerb von Leistungsrechten im System der Gesundheitsfürsorge abgesprochen wird, da es sich hierbei nicht etwa um eine individualisierte Fürsorge für den Fall einer konkreten Gesundheitsbeeinträchtigung handelt, sondern allgemein um die Aufnahme in ein System der Gesundheitsfürsorge und die Erteilung einer generellen Garantie einer solchen Fürsorge in einem zeitlichen und sachlichen Umfang, der sich nach den Umständen des Einzelfalls bestimmen lässt. Aus diesem Grund kann vielmehr davon ausgegangen werden, dass die Feststellung eines Verschuldens auf Seiten des Versicherten zum Verlust oder zur Einschränkung seiner Berechtigung zur Einforderung öffentlich finanzierter Leistungen im System der Gesundheitsfürsorge führt. Dieser Rechtsverlust kann jedoch nicht dahingehend verstanden werden, dass die Erteilung dringender Leistungen einer gegebenenfalls notwendigen Gesundheitsfürsorge schlicht versagt wird. Die hier vorgeschlagene Sanktionierung eines vom Versicherten selbstverschuldeten Gesundheitsverlusts würde stattdessen auf die Verpflichtung einer solchen Person zur selbstständigen Tragung der Kosten - in vollem Umfang oder anteilig - der ihr erteilten Leistungen hinauslaufen. Mit anderen Worten erhielte der Behandlungsbedürftige die notwendige Fürsorge nach den allgemeinen Grundsätzen (Besuch beim Hausarzt, etwaige Untersuchungen, Überweisungen an Fachärzte usw., bzw. Leistungen der Notfallmedizin), jedoch stünde dem Versicherer im Falle der Feststellung einer vorsätzlichen Herbeiführung oder Mitverursachung der Gesundheitsschädigung und der Verneinung eines Zufallsereignisses im Nachhinein ein Regressanspruch zu. ${ }^{61}$

61 Vorbehaltlich der kontroversen Natur dieser These sollte angemerkt werden, dass der Nationale Gesundheitsfonds jetzt schon über Möglichkeiten verfügt, Regressforderungen gegen Verursacher von Situationen, die eine Notwendigkeit der Erteilung medizinischer Leistungen an Versicherte nach sich zogen, nachzugehen, da gem. Art. 116 Abs. 1 Pkt. 6 LÖffGG zu den Einnahmen des Fonds u.a. aus Regressansprüchen gewonnene Mittel zählen. Eingehend zur Problematik vgl. Lach, Roszczenia regresowe przysługujące NFZ, Praca i Zabezpieczenie Społeczne 2005, Heft 8, S. 20-25. 
